\title{
Near Kohn anomalies in the phonon dispersion relations of lead chalcogenides
}

\author{
Ondrej Kilian, ${ }^{1,2}$ Guy Allan, ${ }^{2}$ and Ludger Wirtz ${ }^{2}$ \\ ${ }^{1}$ Department of Astronomy, Physics of the Earth, and Meteorology, Comenius University, \\ Mlynska dolina F1, 84248 Bratislava 4, Slovakia \\ ${ }^{2}$ Institute for Electronics, Microelectronics, and Nanotechnology (IEMN), Department ISEN, CNRS-UMR 8520, B.P. 60069, \\ 59652 Villeneuve d'Ascq Cedex, France
}

(Received 18 September 2008; revised manuscript received 12 September 2009; published 11 December 2009)

\begin{abstract}
We present $a b$ initio phonon dispersion relations for the three lead chalcogenides $\mathrm{PbS}, \mathrm{PbSe}$, and $\mathrm{PbTe}$. The acoustic branches are in very good agreement with inelastic neutron-scattering data and calculations of the specific heat give good agreement with experimental data. The pronounced minimum of the transverse-optical branch at $\Gamma$ due to the near ferroelectricity of the lead chalcogenides is qualitatively reproduced. In addition, we find a pronounced dip in the longitudinal-optical branch at $\Gamma$. This dip was previously explained as the effect of "free carriers" (due to the presence of impurities). The calculations demonstrate that it persists also in the case of pure lead chalcogenides. We explain the dip as a "near Kohn anomaly" which is associated with the small electronic band gap at the high-symmetry point $\mathrm{L}$.
\end{abstract}

DOI: $10.1103 /$ PhysRevB.80.245208

PACS number(s): 63.20.dk, 65.40.Ba

\section{INTRODUCTION}

Lead chalcogenides ( $\mathrm{PbS}, \mathrm{PbSe}$, and $\mathrm{PbTe}$ ) are IV-VI narrow gap semiconductor compounds with rock-salt crystal structure. In nanocrystalline form these materials manifest superior optical and electrical properties which opens a wide field of applications. Their small gap (280-410 meV at room temperature ${ }^{1}$ ), a large exciton diameter [e.g., $20 \mathrm{~nm}$ in $\mathrm{PbS}$ and $46 \mathrm{~nm}$ in PbSe (Ref. 2)] and small effective electron and hole masses make them a good medium for optoelectronics, photovoltaic devices, and quantum confinement studies., ${ }^{2,3}$ An infrared diode laser was constructed ${ }^{4}$ based on $\mathrm{PbSe} /$ $\mathrm{PbEuTe}$ quantum dots. Recent studies ${ }^{5}$ indicate that $\mathrm{PbSe}$ nanocrystals might be good candidates for high-efficient solar cells. Because of impact ionization (an electron-hole pair with large energy decays into several electron-hole pairs with lower energy), efficient carrier multiplication occurs.

In order to fully understand the de-excitation of hot carriers - in particular, the ratio of radiative versus nonradiative decay channels and the mechanisms of line broadening - it is necessary to learn more about the electronphonon coupling in lead chalcogenides. While high-quality calculations of the electronic bands are available (Ref. 6 and references therein), the understanding of the phonon dispersion of the lead chalcogenides is much less complete. Experimentally, phonon dispersion relations of $\mathrm{PbS}, \mathrm{PbSe}$, and $\mathrm{PbTe}$ were obtained by inelastic neutron-scattering measurements. ${ }^{7-9}$ Simulations of the phonon dispersions have been done so far mainly on the level of the semiempirical shell model. ${ }^{7-9,12,13}$ All three materials exhibit the same anomalies in the dispersion relation. A strong softening of the transverse optical (TO) phonon branch around $\Gamma$ and an unexpected dip of the longitudinal optical (LO) branch at $\Gamma$. The TO softening is due to the near-ferroelectric character of the lead chalcogenides ${ }^{10}$ (in a truly ferroelectric material, this mode would acquire a complex frequency, i.e., the fcc structure would no longer be the most stable one). Different explanations were proposed for the LO dip. Cowley and Dolling ${ }^{11}$ attributed the dip to screening by free carriers in the crystal. A term for free-carrier doping was consequently introduced in the recent semiempirical phonon calculations by Upadhyaya et al. ${ }^{12}$ Maksimenko and Mishchenko ${ }^{13}$ explain the LO dip by the dipolar pseudo-Jahn-Teller effect. ${ }^{14}$ Recent $a b$ initio calculations of the phonon dispersions of $\mathrm{PbS}, \mathrm{PbSe}$, and $\mathrm{PbTe}$ (Ref. 15) displayed a minimum of the $\mathrm{LO}$ mode at $\Gamma$ which (for $\mathrm{PbSe}$ and $\mathrm{PbTe}$ ) turned into a strongly pronounced dip when spin-orbit coupling was taken into account. The reason for the strong dip enhancement remained open. The strong stiffening with pressure of the TO mode in PbTe was calculated ab initio by An et al. ${ }^{16}$

We present in this paper a systematic $a b$ initio study of the three phonon dispersion relations of the lead chalcogenides. The small gap and the near-ferroelectric behavior demand a careful choice of calculation parameters such as sampling grid and pseudopotentials. Our calculations reproduce quantitatively the acoustic modes. The anomalies of LO and TO modes are qualitatively reproduced in the calculations (since they are strongly temperature dependent, ${ }^{13}$ anharmonic effects would have to be taken into account in order to quantitatively reproduce the measurements which were performed at room temperature). Our calculations show that a pronounced LO dip is present in the pure materials even without free-carrier doping. We explain this dip in analogy to the Kohn anomalies ${ }^{17}$ that occur in the semimetal graphene. ${ }^{18}$ Furthermore, we demonstrate that ab initio calculations can reproduce very well the specific heat of $\mathrm{PbS}$ and compare with recent calculations ${ }^{15,19}$ that include the effect of spinorbit effects.

In Sec. II we summarize the computation method and give the details of the calculations. In Sec. III we discuss the dispersion relations of the three lead chalcogenides. Section IV presents data on the specific heat in comparison with experimental data. In the Appendix, we show that the electronic $k$-point sampling must be dense enough around the high-symmetry point $\mathrm{L}$ in order to reproduce the LO dip at $\Gamma$.

\section{COMPUTATIONAL DETAILS}

In the harmonic approximation, the phonon frequencies (as a function of the wave vector $\mathbf{q}$ ) are obtained from the equation 
TABLE I. Calculated lattice constants in comparison with experimental lattice constants (Ref. 1) at 30 and $300 \mathrm{~K}$.

\begin{tabular}{lccc}
\hline \hline & $\begin{array}{c}\text { DFT-LDA } \\
(\AA)\end{array}$ & $\begin{array}{c}\text { Expt. (30 K) } \\
(\AA)\end{array}$ & $\begin{array}{c}\text { Expt. (300 K) } \\
(\AA)\end{array}$ \\
\hline $\mathrm{PbS}$ & 5.810 & 5.909 & 5.936 \\
$\mathrm{PbSe}$ & 6.012 & 6.098 & 6.124 \\
$\mathrm{PbTe}$ & 6.318 & 6.428 & 6.462 \\
\hline \hline
\end{tabular}

$$
\operatorname{det}\left|\frac{1}{\sqrt{M_{s} M_{t}}} \widetilde{C}_{s t}^{\alpha \beta}(\mathbf{q})-\omega^{2}(\mathbf{q})\right|=0 .
$$

The dynamical matrix $\widetilde{C}_{s t}^{\alpha \beta}(\mathbf{q})$ corresponds to the force on atom $t$ in direction $\beta$ linearly induced by a displacement of atom $s$ in direction $\alpha$. We calculate it with density-functional perturbation theory ${ }^{20,21}$ as implemented in the code ABINIT. ${ }^{22}$ We use the local-density approximation (LDA) for the exchange-correlation functional. ${ }^{23}$ The wave functions are expanded in plane waves. Core electrons are replaced by pseudopotentials.

We found that the phonon frequencies are very sensitive to the choice of the lead pseudopotential. In particular, it is important to include the lead $5 d$ semicore electrons as valence electrons in the calculation. (This is different from the case of pure lead, where the $5 d$ electrons do not alter the phonon frequencies significantly ${ }^{24}$ ). The reason lies in the strongly ionic character of the lead chalcogenides; the lead atoms tend to transfer the $6 p$ valence electrons to the anions. It is then the overlap of the remaining $6 s$ and $5 d$ electrons of lead with the $3 p$ orbitals of the anion that determines the covalent part of the $\mathrm{Pb} X$ bond (where $X$ stands for the anion $\mathrm{S}, \mathrm{Se}$, or Te, respectively). Since the $5 d$ orbitals contribute to this bonding, their density should be calculated explicitly and not be substituted by a pseudopotential. We tested different Troullier-Martins pseudopotentials created with the FHI pseudopotential generation code. ${ }^{25}$ We verified that the corresponding FHI potential for lead and the chalcogens from the ABINIT webpage yielded converged results for the phonon frequencies. The plane-wave energy cutoff is 40 hartree.

It has been observed for ferroelectric materials that $a b$ initio phonon calculations give better agreement with experimental data if they are performed at the experimental lattice constant, rather than the lattice constant obtained by totalenergy minimization (see Ref. 26 and references therein). The reason is that the LDA tends to underestimate the lattice constant and in (near) ferroelectric materials even a small underestimation of $1 \%$ strongly influences the ferroelectric instability. Our phonon calculations are performed using the experimental lattice constants at $300 \mathrm{~K}$. The experimental lattice constant are given in Table I together with the values of the optimized lattice constants. Since anharmonic effects are neglected (and very difficult to include on an $a b$ initio level ${ }^{27}$ ), we do not expect to fully reproduce the temperature dependence of the phonon dispersions.

The influence of the electronic $k$-point sampling on the phonon dispersion is discussed in the Appendix. For converged results, we used a $(7,3)$ nested grid, i.e., a $7 \times 7 \times 7$
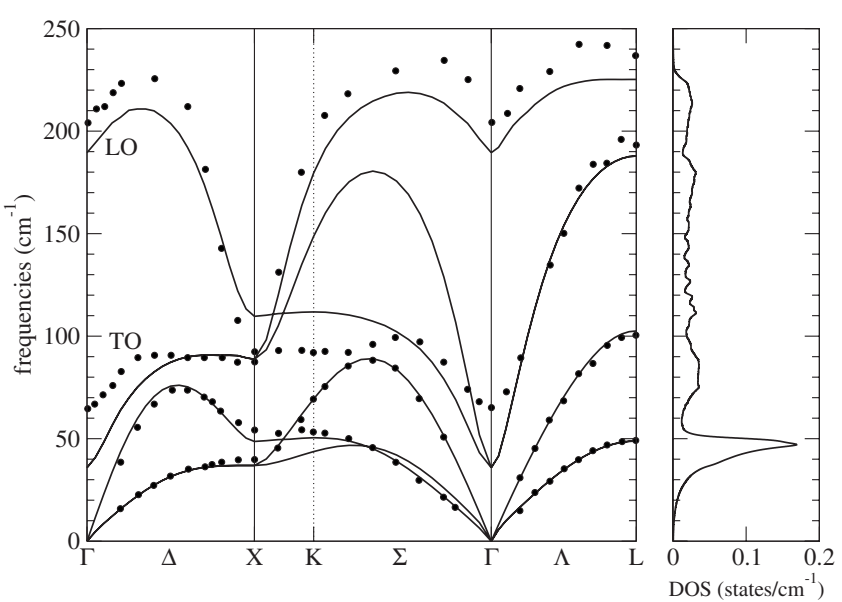

FIG. 1. Left panel: Calculated phonon dispersion relation of lead sulfide (lines) in comparison with experimental data (Ref. 8) (dots). Right panel: phonon density of states.

(shifted) Monkhorst-Pack (MP) $k$-point sampling with an additional $3 \times 3 \times 3$ sampling of the volume element around the high-symmetry point L. In order to obtain the phonons at arbitrary phonon wave vector $\mathbf{q}$, the dynamical matrix $\widetilde{C}_{s t}^{\alpha \beta}(\mathbf{q})$ is calculated on a $8 \times 8 \times 8$ mesh and then Fourier interpolated for arbitrary $\mathbf{q}$. In order to properly reproduce the LO dip around $\Gamma$, we calculated the dynamical matrix explicitly for a set of $\mathbf{q}$ points along the high-symmetry lines $\Delta, \Sigma$, and $\Lambda$ close to the $\Gamma$ point.

\section{PHONON DISPERSION RELATIONS}

In Figs. 1-3, we present our calculated dispersion relations for $\mathrm{PbS}, \mathrm{PbSe}$, and $\mathrm{PbTe}$, respectively, and compare with experimental data from inelastic neutron scattering. We also show the calculated phonon densities of states which will be needed for the calculation of the specific heat. In all three cases, we obtain excellent agreement between theory and experiment for the three acoustic modes. Since the mass of $\mathrm{Pb}$ atoms is much larger than the masses of $\mathrm{S}, \mathrm{Se}$, and $\mathrm{Te}$,
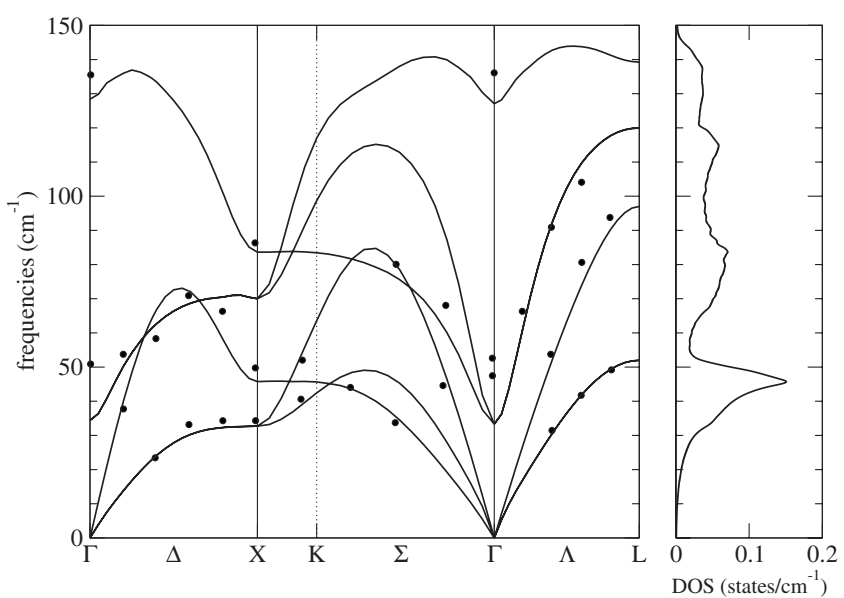

FIG. 2. Calculated phonon dispersion relation of lead selenide (lines) in comparison with experimental data (Ref. 9) (dots). 

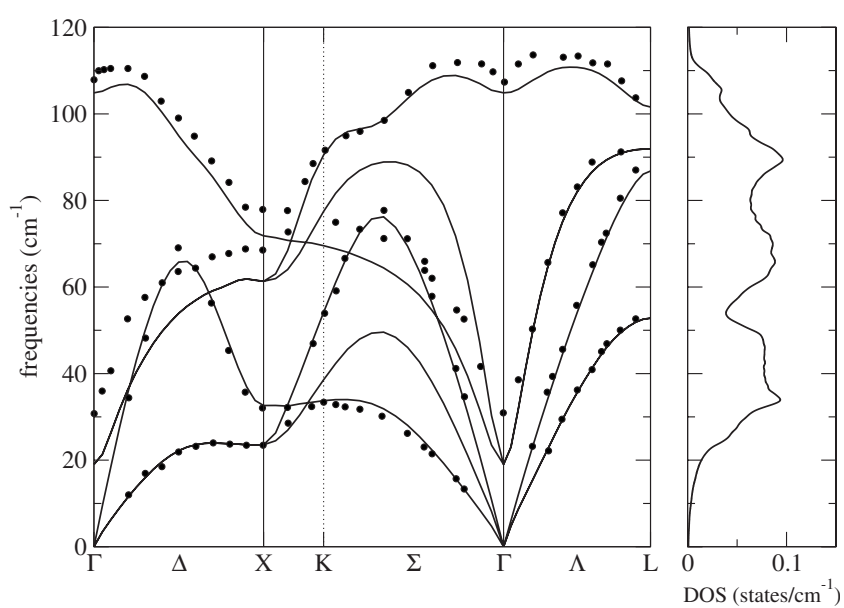

FIG. 3. Calculated phonon dispersion relation of lead telluride (lines) in comparison with experimental data (Ref. 7) (dots).

the acoustic branches correspond almost exclusively to vibrations of lead ions. Consequently, the acoustic-mode dispersion is quantitatively very similar in the three lead chalcogenides. The main difference is a small frequency lowering that corresponds to increasing lattice constant in the series $\mathrm{PbS}, \mathrm{PbSe}$, and $\mathrm{PbTe}$. The three optical branches correspond to vibrations of the anions. Their maximum frequency scales as $1 / \sqrt{M_{X}}$, where $X$ denotes the anion species. For PbS, the optical modes are so high in energy that they do not intersect with the acoustic modes. For PbSe and $\mathrm{PbTe}$ some crossings occur.

In all three cases, we observe a remarkably large LO/TO splitting at $\Gamma$. For cubic systems, the splitting is given by ${ }^{21}$

$$
\omega_{\mathrm{LO}}^{2}-\omega_{\mathrm{TO}}^{2}=\frac{4 \pi e^{2}}{\mu \Omega} \frac{\left(Z^{*}\right)^{2}}{\epsilon},
$$

where $Z^{*}$ is the effective charge, $\epsilon$ is the dielectric constant, $e$ is the electron charge, $\Omega$ is the unit-cell volume, and $\mu$ $=M_{\mathrm{Pb}} M_{X} /\left(M_{\mathrm{Pb}}+M_{X}\right)$ is the effective mass. The values for $Z^{*}$ and $\epsilon$ are given in Table II. In spite of the large dielectric screening, the LO/TO splitting is strongly pronounced due to the very large effective charges. Consequently, the TO mode at $\Gamma$ has a very low frequency. This frequency depends sensitively on the lattice constant. E.g., for PbSe, an artificial increase in the lattice constant by $0.1 \AA$ leads to a softening of the TO-mode frequency below 0 , i.e., toward imaginary values which means that the fcc phase would no longer be the stable one. This is a clear manifestation of the near-

TABLE II. Values of the dielectric constants and the effective charges of the three lead chalcogenides. Calculations are performed on the DFT-LDA level with the experimental lattice constant at 300 $\mathrm{K}$.

\begin{tabular}{lcc}
\hline \hline & $\epsilon$ & $Z^{*}$ \\
\hline $\mathrm{PbS}$ & 19.6 & \pm 4.5 \\
$\mathrm{PbSe}$ & 24.1 & \pm 4.9 \\
$\mathrm{PbTe}$ & 31.7 & \pm 6.1 \\
\hline \hline
\end{tabular}

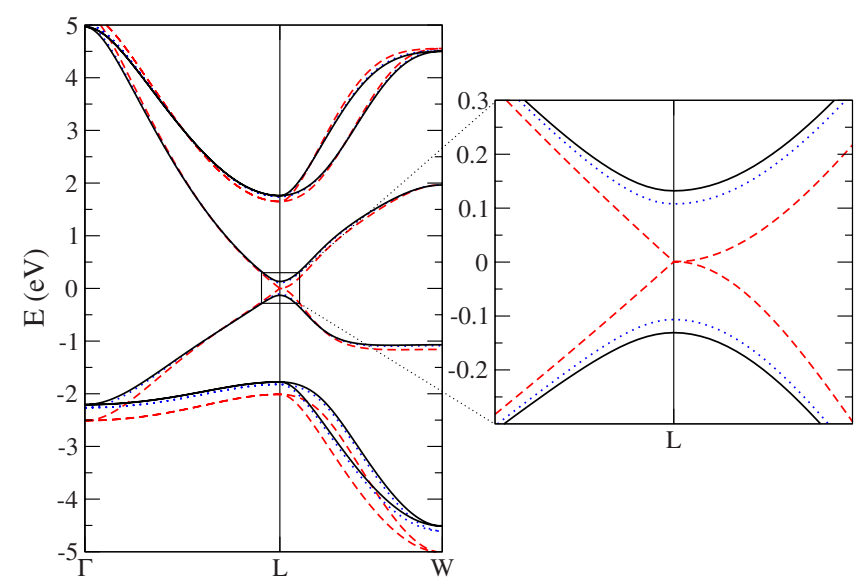

FIG. 4. (Color online) Left panel: Electronic band structure (DFT-LDA without spin-orbit coupling) of PbS for three different values of the lattice constant $a$. Black solid lines: experimental lattice constant at $300 \mathrm{~K}$; blue dotted lines: experimental lattice constant at $30 \mathrm{~K}$; red dashed lines: $a=5.801 \AA$ (squeezed lattice constant which reduces the gap to zero). The Fermi level is at $0 \mathrm{eV}$. A zoom for the region around the direct gap at $\mathrm{L}$ is presented in the right panel.

ferroelectric character of the lead chalcogenides.

All three phonon dispersions exhibit a significant frequency drop of the LO mode at $\Gamma$. Cowley and Dolling ${ }^{11}$ proposed that this phenomenon is caused by the screening of the macroscopic electric field accompanying the LO mode by free carriers (which may have their origin by doping from impurities). We have done our calculations without the presence of additional free carriers, yet we have reproduced the LO anomaly. Free-carrier doping can contribute to the LO dip but seems not to be its primary cause. Our calculations are in accordance with the theory of Maksimenko and Mischenko ${ }^{13}$ who explained the LO anomaly as due to a strong electron-phonon interaction of pseudo-Jahn-Teller type (in absence of free-carrier doping).

We explain this dip as a "near Kohn anomaly." The notion of a Kohn anomaly ${ }^{17}$ is known from metallic systems; the vibrations of the ionic cores are partially screened by the surrounding electron gas. The screening can be strongly enhanced for vibrations with a wave vector $\mathbf{q}$ that connects two points on the Fermi surface. The enhanced screening then leads to a dip in the phonon dispersion at those values of $\mathbf{q}$. Recently, two Kohn anomalies were found in the semimetal (or "zero-gap semiconductor") graphene. ${ }^{18}$ The band structure in the first Brillouin zone of graphene displays two conical intersections (linear crossings) of the $\pi$ and $\pi^{*}$ bands at the Fermi level. The Fermi surface is thus reduced to two points and Kohn anomalies can be found at $\Gamma(\mathbf{q}=0)$ and at K.

The lead chalcogenides are semiconductors. Thus there are-a priori-no Kohn anomalies in their phonon dispersions. However, the direct band gap at the high-symmetry point $\mathrm{L}$ is small and, by compressing the lattice, it can be brought down to zero. The situation is demonstrated for the case of $\mathrm{PbS}$ in Fig. 4. We show the band structure along the high-symmetry lines $\Gamma \rightarrow \mathrm{L} \rightarrow \mathrm{W}$ for three different values of the lattice constant. The highest valence band around $\mathrm{L}$ is 


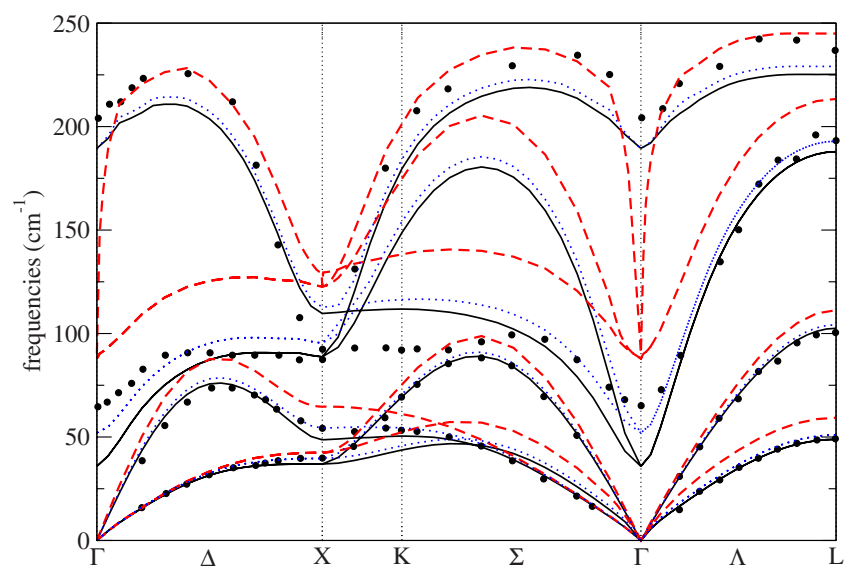

FIG. 5. (Color online) Calculated phonon dispersion of $\mathrm{PbS}$ for three different values of the lattice constant $a$. Black solid lines: experimental lattice constant at $300 \mathrm{~K}$; blue dotted lines: experimental lattice constant at $30 \mathrm{~K}$; red dashed lines: $a=5.801 \AA$ (squeezed lattice constant which reduces the gap to zero). Circles: experimental data (Ref. 8).

composed of S $3 p$ orbitals. The lowest conduction band has a $\mathrm{Pb} 6 p$ character with an admixture of $\mathrm{S} 4 s$ orbitals. When calculated with the experimental lattice constant at $300 \mathrm{~K}$, the LDA-density-functional theory (DFT) band gap is 267 $\mathrm{meV}$. It decreases to the value of $216 \mathrm{meV}$ for the $30 \mathrm{~K}$ experimental lattice constant. Artificially decreasing the lattice constant further reduces the band gap at L. Eventually, for a value of the lattice constant $a=5.801 \AA$, the band gap becomes zero with a linear crossing of the bands in the direction $\mathrm{L} \rightarrow \Gamma$ and a parabolic dispersion in the direction $\mathrm{L}$ $\rightarrow \mathrm{W}$ (see right panel of Fig. 4). This situation is now quite analog to the situation in graphene; the system is semimetallic and the Fermi surface is pointlike, located at the highsymmetry points L. For the corresponding phonon dispersion relation one can expect a Kohn anomaly at $\Gamma$ and at $X$ (the wave-vector difference between to different points $\mathrm{L}$ corresponds to either $\Gamma$ or $X$ ). We note in passing that a further reduction in the lattice constants reopens the gap at L. However, the character of valence and conduction bands is inverted and a real crossing of valence and conduction bands along the line $\mathrm{L} \rightarrow \mathrm{W}$ shows up.

Figure 5 presents the phonon dispersion relations of $\mathrm{PbS}$ for the three different values of the lattice constant. As expected, shrinking the lattice constant leads to a stiffening of the bonds and thus to an increase in the phonon frequencies. In particular, the TO mode is strongly affected because shrinking the lattice constant makes the system "less ferroelectric." The only mode which softens is the highest optical branch at $\Gamma$ and at $X$ where we observe to very sharp dips which correspond to the Kohn anomalies predicted above. Furthermore, LO and TO modes at $\Gamma$ are degenerate for the squeezed lattice; $\epsilon$ tends to infinity in the limit of the semimetallic squeezed lattice and the LO/TO splitting tends to zero according to Eq. (2). Opening the electronic gap by enlarging the lattice constant to its original value reinstalls the LO/TO splitting. However, a remnant of the Kohn anomaly remains visible in the form of a strong dip of the LO mode at $\Gamma$. For this reason, we interpret the LO dip as a near Kohn anomaly.
TABLE III. Values of the band gaps of the lead chalcogenides. Calculations are performed on the DFT-LDA level with the experimental lattice constant at $300 \mathrm{~K}$. Experimental data at $300 \mathrm{~K}$ (Ref. 1).

\begin{tabular}{lcc}
\hline \hline & $\begin{array}{c}E^{\text {gap }}(\text { calc. } \\
(\mathrm{meV})\end{array}$ & $\begin{array}{c}E^{\text {gap }} \text { (expt.) } \\
(\mathrm{meV})\end{array}$ \\
\hline $\mathrm{PbS}$ & 267 & 410 \\
$\mathrm{PbSe}$ & 244 & 280 \\
$\mathrm{PbTe}$ & 644 & 310 \\
\hline \hline
\end{tabular}

We note that the LO dip is considerably less pronounced in our calculated phonon dispersion of PbTe. This is due to the strong overestimation of the gap of this material ${ }^{28}$ (see Table III for our values obtained on the DFT-LDA level and a comparison with the experimental values). At this point, we have to discuss if the inclusion of spin-orbit coupling (SOC) leads to an improvement of the phonon calculations. It has been shown by Hummer et al. ${ }^{6}$ that SOC strongly reduces the electronic gap for the three lead chalcogenides. E.g., for $\mathrm{PbTe}$, the gap is reduced to $60 \mathrm{meV}$. The inclusion of electron-correlation effects, e.g., on the level of the GW approximation, is needed in order to "reopen" the gap and to obtain values in good agreement with the experimental gaps. ${ }^{6}$ For the calculation of the LO dip this means that inclusion of SOC does not necessarily yield better results (unless electron-correlation effects are properly taken care of at the same time); the underestimation of the gap leads to an overestimation of the LO dip. Such an effect can be seen, e.g., in the PbTe phonon dispersion of Romero et al. (Fig. 3 of Ref. 15).

A recent $a b$ initio calculation of Cardona et al. ${ }^{15,19}$ yielded considerably higher phonon frequencies for $\mathrm{PbS}$ than the experimental data. This is due to two reasons: they use the optimized lattice constant $a=5.808 \AA$ which underestimates the experimental lattice constant at $30 \mathrm{~K}$ by $1.7 \%$ and the room-temperature lattice constant by $2.2 \%$. Furthermore, their use of a pseudopotential with the lead $5 d$ electrons in the core may lead to different phonon frequencies. Including SOC, leads to a general softening of the phonons and improves the agreement with experimental data ${ }^{15}$ (except for the LO dip at $\Gamma$ ). Our calculations with the experimental lattice constant at $300 \mathrm{~K}$ are in very good agreement with the acoustic branches of the experimental dispersions. Inclusion of SOC would probably lead to less agreement.

The optical phonons are in worse quantitative agreement with the experimental data points than the acoustic modes. In particular, for the TO mode around $\Gamma$, strong deviations occur. This is not surprising, since our calculations take into account temperature effects only through the choice of the room-temperature lattice constant while phonon renormalization through phonon-phonon interaction ${ }^{27}$ is neglected within the harmonic approximation. Due to the near ferroelectricity, the renormalization of the TO mode as a function of temperature will be particularly strong. E.g., the model calculation by Maksimenko and Mischenko ${ }^{13}$ predicts for PbTe that the TO mode at room temperature stiffens by about $15 \mathrm{~cm}^{-1}$ with respect to its value at $4 \mathrm{~K}$. 

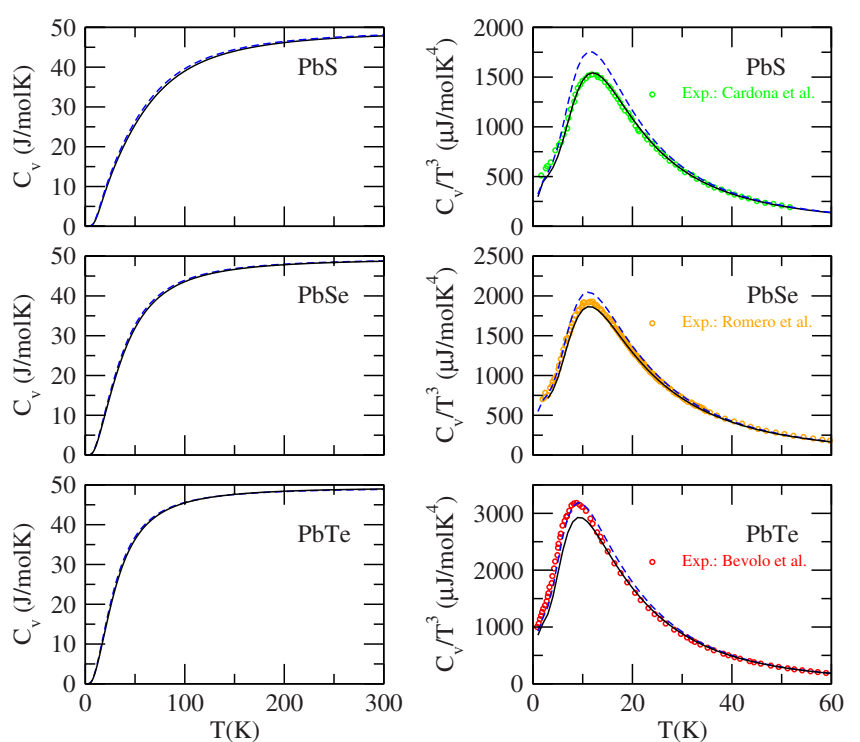

FIG. 6. (Color online) Left panels: Specific heat of the three lead chalcogenides as a function of temperature. Right panels: Specific heat divided by $T^{3}$ in the low-temperature regime. Results from $a b$ initio phonon calculations using the respective lattice constants at $30 \mathrm{~K}$ (solid line) and at $300 \mathrm{~K}$ (dashed lines). Symbols: Experimental data from Ref. 19 (Cardona et al.), Ref. 15 (Romero et al.), and Ref. 30 (Bevolo et al.).

\section{SPECIFIC HEAT}

Another test of the quality of our ab initio phonon calculations is the comparison with available experimental data for the specific heat, $c_{v}$, of $\mathrm{PbS}, \mathrm{PbSe}$, and PbTe. ${ }^{15,19,29,30}$ The specific heat depends on the phonon densities of states, $D(\omega)$, (right panels of Figs. 1-3). We calculate it numerically through the formula

$$
c_{v}=N_{A} k \int_{0}^{\infty} \frac{\left(\frac{\hbar \omega}{k T}\right)^{2} e^{\hbar \omega / k T}}{\left(e^{\hbar \omega / k T}-1\right)^{2}} D(\omega) d \omega,
$$

where $k$ is the Boltzmann constant and $N_{A}$ is the Avogadro constant. Note that $D(\omega)$ is normalized to 6 , i.e., the number of phonon branches.

The resulting specific heat as a function of temperature is plotted in the left panels of Fig. 6. All three curves display the typical convergence toward the Petit and Dulong value $6 N_{A} k=49.9 \mathrm{~J} / \mathrm{mol} \mathrm{K}$ for a material with two atoms in the primitive cell. Following the discussion of Cardona et al. in Refs. 15 and 19, we also display $c_{v} / T^{3}$ in the lowtemperature regime (right panels of Fig. 6). All three curves display a maximum between 8 and $12 \mathrm{~K}$.

The agreement between the experimental and theoretical height of the maximum of $c_{v} / T^{3}$ was used in Ref. 19 as a critical test for the quality of the $a b$ initio phonon calculations. For $\mathrm{PbS}$, they obtained a maximum height of the $a b$ initio curve at $1160 \mu \mathrm{J} / \mathrm{mol} \mathrm{K}^{4}$ while the experimental height is at $1520 \mu \mathrm{J} / \mathrm{mol} \mathrm{K}^{4}$. The deviation was tentatively assigned to the absence of SOC in the calculations. Recently, it was shown for elemental bismuth ${ }^{31}$ and antimony ${ }^{32}$ that inclusion of SOC in the phonon calculations leads to a lowering of the acoustic modes and thus to an increase in the maximum $c_{v} / T^{3}$. Also for elemental lead, a lowering of the acoustic modes through the inclusion of spin-orbit coupling has been observed. ${ }^{24}$

Since our dispersion relations have been calculated with the room-temperature lattice constants, we expect that we underestimate the frequencies of the acoustic phonons at very low temperature (where the lattice constant shrinks and the interatomic force constants stiffen). Consequently, our computed $c_{v} / T^{3}$ for $\mathrm{PbS}$ should overestimate the measured one. Figure 6 (blue dashed line) shows that this is indeed the case; we obtain the maximum $c_{v} / T^{3}$ at $1750 \mu \mathrm{J} / \mathrm{mol} \mathrm{K}^{4}$. For a better assessment of the specific heat at low temperature, we have repeated the calculation of the phonon dispersion and the density of states (DOS) using the PbS lattice constant at $30 \mathrm{~K}$ (see Table I). The resulting $c_{v} / T^{3}$ (solid black line in Fig. 6) is in excellent agreement with the measured data. For $\mathrm{PbSe}$, the agreement with experiment is also fairly good. However, in the case of PbTe, the specific heat calculated with the phonons at the low-temperature lattice constant is somewhat lower than the experimental data. Consequently, spin-orbit coupling [which has a stronger effect in PbTe than in $\mathrm{PbSe}$ and $\mathrm{PbS}$ (Ref. 15)] might be needed to yield good agreement with the experimental data. Further calculations of the phonon DOS including SOC effects (and the $5 d$ electrons of $\mathrm{Pb}$ in the valence) are needed to resolve this issue.

\section{CONCLUSION}

We have calculated the phonon dispersion relations for lead chalcogenides. Strict convergence parameters (concerning the lead pseudopotential and the $k$-point sampling) enabled us to obtain good agreement with experimental dispersion relations and measurements of the specific heat. The acoustic-phonon modes are reproduced almost exactly. The pronounced dip of the LO mode at $\Gamma$ is related to the narrow band gap and can be understood as a near Kohn anomaly. This work provides the starting point for the investigation of electron-phonon coupling in nanocrystals of lead chalcogenides.

\section{ACKNOWLEDGMENTS}

We would like to thank C. Delerue, J. Serrano, M. Cardona, A. Romero, X. Gonze, and M. Verstraete for stimulating discussions. Funding was provided by the French $\mathrm{Na}$ tional Research Agency through Project No. ANR PJC05_6741. Calculations were done at the IDRIS supercomputing center, Orsay (Project No. 081827).

\section{APPENDIX: INFLUENCE OF THE $\boldsymbol{k}$-POINT SAMPLING ON THE LO-MODE FREQUENCY AT $\Gamma$}

For most semiconductors, a $4 \times 4 \times 4$ or $6 \times 6 \times 6$ (shifted) Monkhorst-Pack $k$-point sampling ${ }^{33}$ of the electronic structure is sufficient to reproduce the phonon dispersion, including the LO/TO splitting at $\Gamma$ for polar materials. In this appendix, we show that for lead chalcogenides a 

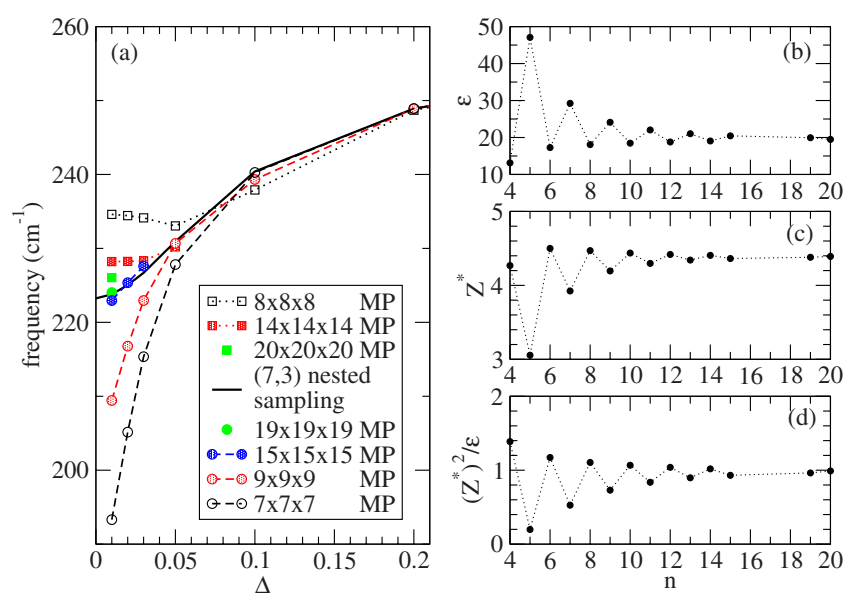

FIG. 7. (Color online) (a) LO-mode dispersion in PbS for different MP samplings and a nested sampling (see text). The dispersion is plotted along the line $\Delta(\Gamma \rightarrow X)$ with 0 corresponding to $\Gamma$ and 0.5 corresponding to $X$. (b) dielectric function $\epsilon$ as a function of $n$, where $n \times n \times n$ denotes the Monkhorst-Pack sampling. (c) Effective charge $Z^{*}$ as a function of sampling. (d) $\left(Z^{*}\right)^{2} / \epsilon$ as a function of sampling.

much higher sampling is needed to properly reproduce the LO-mode dispersion around $\Gamma$.

Figure 7(a) shows the LO-mode dispersion of PbS close to $\Gamma$ for different $n \times n \times n$ Monkhorst-Pack samplings. We used here a Troullier-Martins pseudopotential with the $5 d$ electrons in the core (from the ABINIT webpage). This allowed us to go to very high $k$-point samplings. With this pseudopotential, we obtain a gap of $231 \mathrm{meV}$ which is close enough to the converged value of $267 \mathrm{meV}$ (see Table III) to reproduce a similarly strong anomaly of the LO mode. The phonon frequencies marked by the symbols have been obtained by directly calculating the dynamical matrix for the corresponding phonon wave vector (avoiding interpolation of the dynamical matrix). Obviously, it makes a big difference if $n$ is even or odd. For $n$ even, we obtain higher frequencies and for $n$ odd, we obtain lower frequencies than in the limit $n \rightarrow \infty$. The difference between the $7 \times 7 \times 7$ and the
$8 \times 8 \times 8$ sampling amounts to more than $40 \mathrm{~cm}^{-1}$ for the LO mode at $\Gamma$. (For the TO mode-not shown here-the corresponding difference is less than $3 \mathrm{~cm}^{-1}$.) Even between the $19 \times 19 \times 19$ sampling and the $20 \times 20 \times 20$ sampling, there remains a difference of $2 \mathrm{~cm}^{-1}$ for the LO mode (while the TO mode is converged to within $0.03 \mathrm{~cm}^{-1}$ ).

The origin of the even-odd discrepancy for the different $k$-point samplings lies in the electronic structure of the lead chalcogenides which all have a very small direct gap at the high-symmetry point L. The small gap is (among other factors) responsible for the very high dielectric constants $(\epsilon$ $>19$ ) of the lead chalcogenides. The point $\mathrm{L}$ is included in the samplings when $n$ is odd but not when $n$ is even. Figure 7 (b) demonstrates that response-function calculations with odd samplings tend to strongly overestimate $\epsilon$ while the even samplings underestimate it. Similar even-odd oscillations for low $k$-point samplings are observed for the calculated effective charges [panel (c)]. The link between the dielectric screening, effective charges, and the LO/TO splitting is given by Eq. (2). Panel (d) demonstrates that the ratio $\left(Z^{*}\right)^{2} / \epsilon$ also displays the even-odd oscillations and converges slowly with $n$. Obviously, Monkhorst-Pack grids with an even $n$ lead to much faster convergence than grids with odd $n$.

Since a calculation of the full dispersion relation with a very dense Monkhorst-Pack grid (and with the $5 d$ electrons in the valence) was not feasible, we used a nested $(7,3)$ grid. This is a $7 \times 7 \times 7$ MP grid where, in addition, the cubic volume element around the point $\mathrm{L}$ is sampled by a simple $3 \times 3 \times 3$ grid. The high $k$-point density around L corresponds thus to the density in a uniform $21 \times 21 \times 21$ grid and the solid line in Fig. 7(a) demonstrates that we obtain the LO frequency in very good agreement with the $20 \times 20 \times 20$ and $19 \times 19 \times 19$ samplings.

Due to the pronounced dip, the calculation of the dispersion relation from a Fourier-interpolated dynamical matrix is not feasible for the LO mode around $\Gamma$. This is the reason, why in our dispersion relations (Figs. 1-3), we used interpolation for most of the Brillouin zone but added point-bypoint calculations for the $\mathrm{LO}$ mode close to $\Gamma$.
${ }^{1}$ Semiconductors: Group IV Elements, IV-IV and III-IV Compounds, Landolt-Börnstein, New Series, Group III, Vol. 41, Pt. A, edited by O. Madelung, U. Rössler, and M. Schulz (SpringerVerlag, Berlin, 2005).

${ }^{2}$ F. W. Wise, Acc. Chem. Res. 33, 773 (2000).

${ }^{3}$ B. L. Wehrenberg, C. Wang, and P. Guyot-Sionnest, J. Phys. Chem. B 106, 10634 (2002).

${ }^{4}$ G. Springholz, T. Schwarzl, W. Heiss, G. Bauer, M. Aigle, and H. Pascher, Appl. Phys. Lett. 79, 1225 (2001).

${ }^{5}$ R. D. Schaller and V. I. Klimov, Phys. Rev. Lett. 92, 186601 (2004).

${ }^{6}$ K. Hummer, A. Grüneis, and G. Kresse, Phys. Rev. B 75, 195211 (2007).

${ }^{7}$ W. Cochran, R. A. Cowley, G. Dolling, and M. M. Elcombe,
Proc. R. Soc. London, Ser. A 293, 433 (1966).

${ }^{8}$ M. M. Elcombe, Proc. R. Soc. London, Ser. A 300, 210 (1967).

${ }^{9}$ P. R. Vijayraghavan, S. K. Sinha, and P. K. Iyengar, Proc. Nucl. Phys. Solid State Phys. Symp. 16C, 208 (1973).

${ }^{10}$ W. Jantsch, A. Bussmann-Holder, H. Bilz, and P. Vogl, Dynamical Properties of IV-VI Compounds, Springer Tracts in Modern Physics Vol. 99 (Springer, Berlin, 1983).

${ }^{11}$ R. A. Cowley and G. Dolling, Phys. Rev. Lett. 14, 549 (1965).

${ }^{12}$ K. S. Upadhyaya, M. Yadav, and G. K. Uphadhyaya, Phys. Status Solidi B 229, 1129 (2002)

${ }^{13}$ O. B. Maksimenko and A. S. Mishchenko, J. Phys.: Condens. Matter 9, 5561 (1997).

${ }^{14}$ G. A. Gehring and K. A. Gehring, Rep. Prog. Phys. 38, 1 (1975).

${ }^{15}$ A. H. Romero, M. Cardona, R. K. Kremer, R. Lauck, G. Siegle, 
J. Serrano, and X. C. Gonze, Phys. Rev. B 78, 224302 (2008).

${ }^{16}$ J. An, A. Subedi, and D. J. Singh, Solid State Commun. 148, 417 (2008).

${ }^{17}$ W. Kohn, Phys. Rev. Lett. 2, 393 (1959).

${ }^{18}$ S. Piscanec, M. Lazzeri, F. Mauri, A. C. Ferrari, and J. Robertson, Phys. Rev. Lett. 93, 185503 (2004).

${ }^{19}$ M. Cardona, R. K. Kremer, R. Lauck, G. Siegle, J. Serrano, and A. H. Romero, Phys. Rev. B 76, 075211 (2007).

${ }^{20}$ S. Baroni, P. Giannozzi, and A. Testa, Phys. Rev. Lett. 58, 1861 (1987); X. Gonze, Phys. Rev. A 52, 1096 (1995).

${ }^{21}$ S. Baroni, S. de Gironcoli, A. Dal Corso, and P. Giannozzi, Rev. Mod. Phys. 73, 515 (2001).

${ }^{22}$ X. Gonze, J.-M. Beuken, R. Caracas, F. Detraux, M. Fuchs, G.-M. Rignanese, L. Sindic, M. Verstraete, G. Zérah, F. Jollet, M. Torrent, A. Roy, M. Mikami, Ph. Ghosez, J.-Y. Raty, and D. C. Allan, Comput. Mater. Sci. 25, 478 (2002). The ABINIT code results from a common project of the Université Catholique de Louvain, Corning Incorporated, and other collaborators (http:// www.abinit.org).

${ }^{23}$ W. Kohn and L. J. Sham, Phys. Rev. 140, A1133 (1965).
${ }^{24}$ M. J. Verstraete, M. Torrent, F. Jollet, G. Zérah, and X. Gonze, Phys. Rev. B 78, 045119 (2008).

${ }^{25}$ M. Fuchs and M. Scheffler, Comput. Phys. Commun. 119, 67 (1999).

${ }^{26}$ R. Resta, Modell. Simul. Mater. Sci. Eng. 11, R69 (2003).

${ }^{27}$ N. Bonini, M. Lazzeri, N. Marzari, and F. Mauri, Phys. Rev. Lett. 99, 176802 (2007).

${ }^{28}$ In Ref. 6, Table IV, a similar overestimation of the PbTe gap is observed, using the FP-APW+LO approach with the PBE functional.

${ }^{29}$ D. H. Parkinson and J. E. Quarrington, Proc. Phys. Soc., London, Sect. A 67, 569 (1954).

${ }^{30}$ A. J. Bevolo, H. R. Shanks, and D. E. Eckels, Phys. Rev. B 13, 3523 (1976).

${ }^{31}$ L. E. Díaz-Sánchez, A. H. Romero, M. Cardona, R. K. Kremer, and X. Gonze, Phys. Rev. Lett. 99, 165504 (2007).

${ }^{32}$ J. Serrano, R. K. Kremer, M. Cardona, G. Siegle, L. E. DíazSánchez, and A. H. Romero, Phys. Rev. B 77, 054303 (2008).

${ }^{33}$ H. J. Monkhorst and J. D. Pack, Phys. Rev. B 13, 5188 (1976). 